\title{
A SEARCH FOR T TAURI'S OPTICAL COMPANION STAR
}

\author{
P. W. Gorham, ${ }^{1}$ A. M. Ghez, C. A. Haniff, ${ }^{2}$ S. R. Kulkarni, K. Matthews, and G. Neugebauer \\ Division of Physics, Mathematics, and Astronomy, and Palomar Observatory, California Institute of Technology, Pasadena, California 91125 \\ Received 26 July 1991; revised 4 November 1991
}

\begin{abstract}
Speckle interferometric observations of the pre-main-sequence stellar system T Tauri at 650 and 800 $\mathrm{nm}$, and at $1.6(H)$ and $2.2 \mu \mathrm{m}(K)$ in the infrared using the Palomar 200 in. telescope yield no evidence for a tertiary optical companion star as reported in 1985. The present observations were of sufficient sensitivity to have detected the object unambiguously if it had remained at its reported brightness and color. The infrared results provide particularly stringent limits on the presence of a third star in the system, since both the optical and southern infrared component are clearly resolved to the diffraction limit of the 200 in. telescope, including the $H$ band where the southern component appeared $\sim 5$ mag fainter than the optical primary. We discuss possible resolutions to the conflicting results concerning this object, and conclude that if the star is bound to the T Tau system, it has dimmed by at least 1-3 mag at $R, I, H$, and $K$. In addition, we note that the star is unlikely to be a normal $\mathrm{M}$ dwarf as reported, and we discuss the possibility that the star could be a Herbig-Haro object.
\end{abstract}

\section{INTRODUCTION}

The pre-main sequence stellar system $\mathrm{T}$ Tauri contains a 9th magnitude optical primary (denoted here as T Tau O), of approximate visual spectral type $K 0$, and a secondary star ( $\mathrm{T}$ Tau S), located $\sim 0.7$ " south of $\mathrm{T}$ Tau $\mathrm{O}$, which becomes increasingly bright at wavelengths longer than $1 \mu \mathrm{m}$; see Dyck et al. (1982), Schwartz et al. (1984, 1986), and Ghez et al. (1991), and references therein. A third component was reported by Nisenson et al. (1985) to exist 0.27 " to the north of the visual primary. This object, denoted here as T Tau N, was detected using speckle interferometric techniques. $\mathrm{T}$ Tau $\mathrm{N}$ was reported to be significantly fainter than $\mathrm{T}$ Tau $\mathrm{O}$, with magnitude differences with respect to $\mathrm{T}$ Tau $\mathrm{O}$ of 5.3 mag at $520 \mathrm{~nm}$ and $3.5 \mathrm{mag}$ at $650 \mathrm{~nm}$. Nisenson et al. suggested that $\mathrm{T}$ Tau $\mathrm{N}$ was another star with a spectral type in the range $M 4-M 8$, and distinct from the primary visual and infrared components. Using one-dimensional $K$ band speckle measurements made in 1988, Maihara \& Kataza (1991) report a detection of this source.

We have made speckle interferometric observations on the T Tau system at $650 \mathrm{~nm}$, and $800 \mathrm{~nm}$ using the Palomar 200 in. telescope in an effort to independently confirm the existence of a third star in the system. Limits obtained from observations at the $1.6 \mu \mathrm{m}(H)$ and $2.2 \mu \mathrm{m}(K)$ infrared bands are also used. These observations were made to study $\mathrm{T}$ Tau S; the results are presented in a separate paper (Ghez et al. 1991). The estimate by Nisenson et al. (1985) of the very red color of T Tau N prompted us to include the $800 \mathrm{~nm}$ and infrared observations in the search. In no case did we detect a third component anywhere in the range $0.02-1.2$ " of the optical primary. The magnitude limits presented here constrain $\mathrm{T}$ Tau $\mathrm{N}$ in such a way that (a) it must be significantly fainter than reported in 1985 and 1988, due either to intrinsic variability or a change in extinction; or (b) the star was an emission line source such as a Herbig-Haro object; or (c) the star was not bound to the system, appeared by superposition, and has since moved away (or to within 20 mas of the visual primary) through large proper motion. We con-

\footnotetext{
${ }^{1}$ Present address: Department of Physics and Astronomy, University of Hawaii at Manoa, 2505 Correa Rd., Honolulu, HI 96822.

${ }^{2}$ Present address: Institute for Astronomy, Madingly Road, Cambridge, United Kingdom.
}

clude that (a) is the most likely scenario, but (b) is a possibility that we cannot exclude.

\section{OBSERVATIONS AND DATA REDUCTION}

Both optical and infrared observations were made at the $f / 415$ Gregorian focus of the 200 in. telescope at Palomar Observatory. The optical photon-counting camera and associated electronics are described in Nakajima et al. (1989) and Gorham et al. (1989). The scale at the $f / 415$ focus is 0.098 " per $\mathrm{mm}$ and the active area of the detector is $25 \mathrm{~mm}$ in diameter, giving a field of view of $2.45^{\prime \prime}$, and an angular sampling of $\sim 6$ mas per $60 \mu \mathrm{m}$ resolution element on the detector. Interference filters centered at 650 and $800 \mathrm{~nm}$, with bandpasses of 7.4 and $25 \mathrm{~nm}$, respectively, were used. The broader bandpass used at $800 \mathrm{~nm}$ helped to compensate for the low quantum efficiency $(\sim 3 \%)$ of the detector at this wavelength.

The 650 and $800 \mathrm{~nm}$ observations were performed on 10 September 1990. Observations of 4-6 min duration were interleaved with observations of two different calibration stars, SAO 93887 and SAO 93898, near T Tau. The visual seeing during the observations was estimated to be $\sim 1$ " FWHM. During the same observing run, a program to search for companions among bright Wolf-Rayet stars yielded one detection which provides an excellent cross calibration of the sensitivity of the search. The star, WR 138 (HD 193077), was observed both in the red continuum near $700 \mathrm{~nm}$, and later in a narrowband filter centered on the $468.6 \mathrm{~nm}$ He II line, which is strong in emission for WolfRayet stars. A secondary component was detected in both bands, as described in the following section. A more detailed description of the results of the Wolf-Rayet survey will be published separately (Gorham et al. 1992). Table 1 provides a log of the observations, including those of WR 138 and its calibrators.

The relevant infrared observations were made over a time span from 1988 August to 1990 December, and include seven different $H$ and $K$ observations, yielding a total of 21 different images used in the search for T Tau N. The observations were made primarily to study $\mathrm{T}$ Tau $\mathrm{S}$, and include many additional observations spanning the range $1.6-20 \mu \mathrm{m}$ in wavelength, made over a total of 8 yrs in time. These are reported in detail in Ghez et al. (1991). 
TABLE 1. Visual observation log.

\begin{tabular}{|c|c|c|c|c|c|}
\hline Object & Type & $\begin{array}{l}\text { Filter, Width } \\
\text { (nm) }\end{array}$ & $\begin{array}{l}\text { Rate }^{\mathrm{a}} \\
\text { (cts/s) }\end{array}$ & $\begin{array}{l}\text { Duration } \\
\text { (s) }\end{array}$ & UT date \\
\hline $\begin{array}{l}\text { SAO } 69399 \\
\text { WR } 138 \\
\text { SAO } 69727\end{array}$ & $\begin{array}{l}\text { calibrator } \\
\text { source } \\
\text { calibrator }\end{array}$ & $\begin{array}{l}700,7 \\
700,7 \\
700,7\end{array}$ & $\begin{array}{l}29000 \\
18000 \\
43000\end{array}$ & $\begin{array}{l}210 \\
350 \\
140\end{array}$ & $\begin{array}{c}1990 \text { September } 8 \\
\text { - }\end{array}$ \\
\hline $\begin{array}{l}\text { SAO } 69653 \\
\text { WR } 138 \\
\text { SAO } 49524\end{array}$ & $\begin{array}{l}\text { calibrator } \\
\text { source } \\
\text { calibrator }\end{array}$ & $\begin{array}{l}467.7,6 \\
467.7,6 \\
467.7,6\end{array}$ & $\begin{array}{l}26000 \\
10000 \\
19000\end{array}$ & $\begin{array}{l}240 \\
620 \\
330\end{array}$ & $\begin{array}{c}1990 \text { September } 9 \\
\text { - }\end{array}$ \\
\hline $\begin{array}{l}\text { T Tau } \\
\text { SAO } 93898 \\
\text { SAO } 93887 \\
\text { T Tau } \\
\text { T Tau } \\
\text { SAO 93898 } \\
\text { SAO } 93887 \\
\text { T Tau }\end{array}$ & $\begin{array}{l}\text { source } \\
\text { calibrator } \\
\text { calibrator } \\
\text { source } \\
\text { source } \\
\text { calibrator } \\
\text { calibrator } \\
\text { source }\end{array}$ & $\begin{array}{l}650,7.4 \\
650,7.4 \\
650,7.4 \\
650,7.4 \\
800,25 \\
800,25 \\
800,25 \\
800,25\end{array}$ & $\begin{array}{l}20000 \\
26000 \\
42000 \\
20000 \\
15000 \\
16000 \\
24000 \\
15000\end{array}$ & $\begin{array}{l}375 \\
240 \\
150 \\
320 \\
400 \\
310 \\
210 \\
250\end{array}$ & $\begin{array}{c}1990 \text { September } 10 \\
= \\
= \\
= \\
=\end{array}$ \\
\hline
\end{tabular}

${ }^{\mathrm{a}}$ This is the observed rate, uncorrected for deadtime effects. Deadtime corrected rates are 10\%-50\% higher.

It is possible to recover complete diffraction-limited images from speckle interferometric data, rather than autocorrelation functions (ACFs) or the Fourier equivalent power spectra. This may be done by using, for example, techniques of bispectral analysis (cf. Lohmann et al. 1983). However, the recovered images invariably have a lower signal-to-noise ratio than the recovered ACFs when the observations are made in the photon-noise-limited regime, where the average number of detected photons per atmospheric coherence cell per coherence time is less than one. In this regime, which obtains for our optical observations, a speckle interferometric search for additional unresolved components around a star is most sensitive using the ACF (or power spectrum) rather than a recovered image of the source structure.

The data reduction procedure for recovering a diffractionlimited ACF of the source structure is described in Gorham et al. (1990). It makes use of the CLEAN algorithm (Hogbom 1974) to deconvolve the atmospheric seeing effects. As shown in Gorham et al., the technique is robust and has routinely produced signal-to-noise ratios (SNRs), defined as the ratio of the peak value to that of the rms noise in the ACF, of $>200$ in the recovered ACFs of binary star speckle interferometry data obtained with the 200 in. telescope. Since the ACFs reported here have typically of order $10^{3}$ resolution elements in them, the number of samples is sufficient to provide a reasonable estimate of the noise. However, because CLEAN is inherently a nonlinear technique, due to the feedback loop used in subtracting the beam from the local maxima, it is necessary to demonstrate that the SNR that results from applying CLEAN is consistent with that found in the raw data. In fact it is possible for CLEAN to improve the SNR under certain restrictions, for example, when a stationary telescope or optical aberration is convolved with the source. This is analogous to the way CLEAN improves radio interferometer maps, where the sidelobes are caused by the shape of the discrete element aperture that is used, rather than by aberration.

\section{RESULTS}

\subsection{Visual Sensitivity Calibration}

Figure 1(a) shows the recovered $700 \mathrm{~nm} \mathrm{ACF}$ of the Wolf-Rayet star WR 138, with a companion at $\sim 0.8$ " separation. The seeing during this observation was $1.2^{\prime \prime}$ FWHM as measured in the raw ACF; thus the secondary could not be visually resolved. The lowest contours here begin at $\pm 0.4 \%$ of the maximum and $\Delta m=4.3 \pm 0.2 \mathrm{mag}$ between the primary and secondary. Since CLEAN is known to have the shortcoming of tending to find spurious point sources in noise, a highpass-filtered image of the raw ACF was also made to be certain that the component was evident in the raw data. This filtered ACF is shown in Fig. 1(b), in which one-dimensional slices through the ACF are overlain. Again the secondary is clearly evident, though now the noise level due to the telescope point-spread function is significantly higher than in the CLEAN map. The excess noise near the central component is in fact quite stationary and most of it is removed in the deconvolution procedure since it appears equally in the calibration star ACF. The final confirmation of both the presence of the secondary component and the sensitivity of our speckle technique is shown in Fig. 1(c), which is a contour plot of the recovered ACF of the WR 138 system in the He II 468.6 emission band. Here the companion is detected just near the noise level with $\Delta m=5.3 \pm 0.3$ mag. The calibration stars for this observation were somewhat mismatched with the WR star; thus a negative "bowl" is seen at the $2 \%$ level around the central component. For the T Tau observations, the calibration was generally much better, matching the low spatial frequencies to $0.5 \%-1 \%$ as discussed below. Small mismatches of this sort are common in speckle interferometry because of seeing instability. However, at this level they do not significantly reduce the sensitivity to stellar secondary components, since a simple highpass filter can effectively remove the mismatch.

WR 138 is known to have an absorption line system thought to be due to a secondary star (Van der Hucht 1983), but the secondary has not been resolved previously, and it is not clear if the secondary component reported here is sufficiently bright to provide the necessary absorption lines. The star was previously surveyed by Moffat et al. (1985) using speckle interferometric techniques, but the limiting sensitivity of the observations was $\Delta m=2 \mathrm{mag}$, which is insufficient to detect this component, and sets an upper limit on its brightness that is consistent with our detection.

\subsection{Visual T Tau Results}

Figure 2 shows the recovered ACF of T Tau as observed at $800 \mathrm{~nm}$; the cross marks the position of T Tau N in 1984. The SNR for this ACF is $\sim 350$. A diamond marks the position of T Tau S in 1990 from Ghez et al. (1991). There is some 

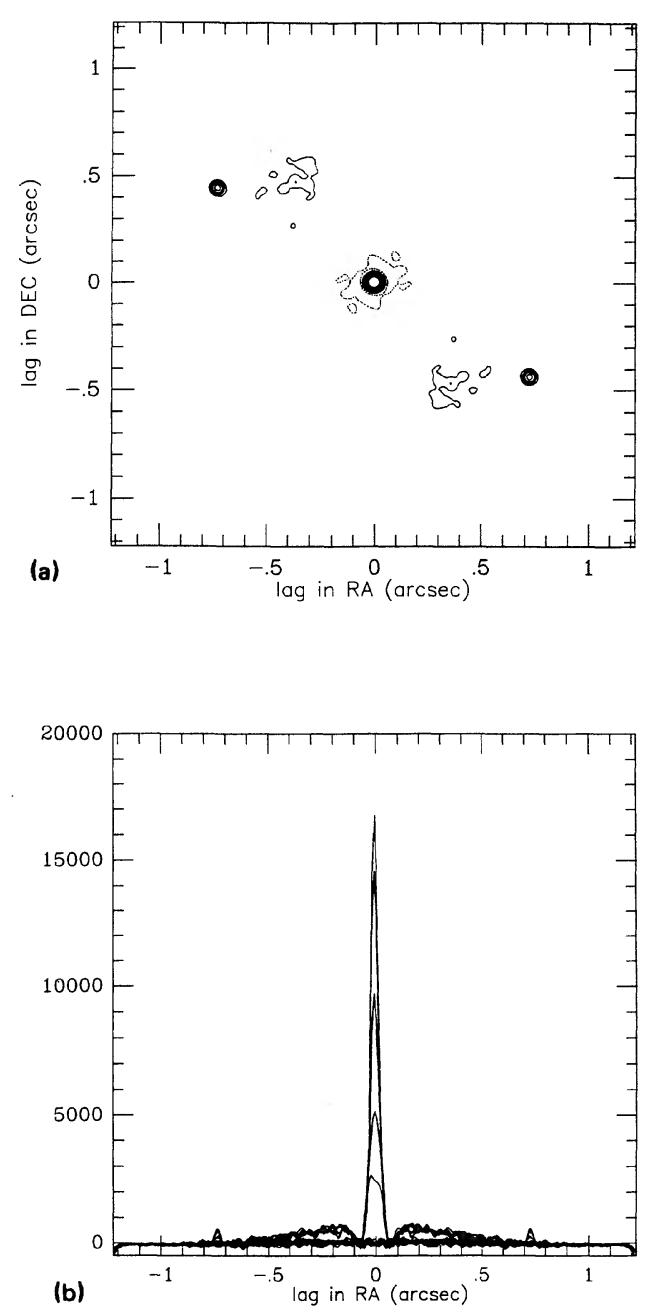

low spatial frequency noise apparent at the $0.5 \%$ level (negative contours). The highest noise maximum appears at a level of $1 \%$ of the central peak.

Figure 3 presents the recovered ACF of T Tau as observed at $650 \mathrm{~nm}$. The SNR of the ACF is $\sim 120$. Here the dominant noise is a low spatial frequency saddle at the $1 \%$ level which is due to residual mismatches of the seeing calibration. To enhance the contrast of this plot for weak stellar components, a high-pass Butterworth filter which passed frequencies above $\sim 3$ cycles per arcsecond was applied to the ACF. The result is shown in Fig. 4, which now has a SNR of 700. In both of these ACFs the central peak shows east-west distortion at the level of $\sim 15$ mas. as in Fig. 1. This appears to be an artifact, and is most likely due to either residual optical aberration, or possible seeing effects.

\subsection{Infrared T Tau Results}

Since the spectral energy distribution of an $\mathbf{M}$ dwarf on the main sequence nominally peaks between the $H$ and $K$ infrared bands, and the resolution of the $200 \mathrm{in}$. telescope is adequate at these wavelengths to easily resolve a pair of stars at $0.3^{\prime \prime}$ separation, the $H$ and $K$ observations were deemed the most relevant in our search. However, since both $T$ Tau

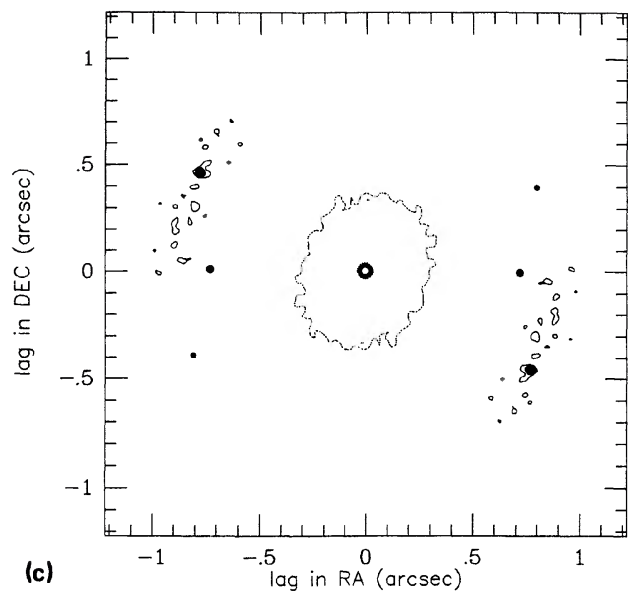

FIG. 1. (a) Recovered ACF of the Wolf-Rayet binary WR 138 at $700 \mathrm{~nm}$, with separation $\sim 0.8$ ". Contours at $\pm 0.4 \%$, $0.6 \%, 0.8 \%, 1.0 \%, 1.5 \%, 2 \%, 4 \%, 8 \%, 16 \%$, and $32 \%$ of the maximum. The magnitude difference is $4.3 \pm 0.2 \mathrm{mag}$, demonstrating the sensitivity of the contemporaneous $\mathrm{T} \mathrm{Tau}$ observations. (b) A set of overlain slices of a highpass-filtered image of the raw ACF of WR 138 at $700 \mathrm{~nm}$, showing that the secondary is quite evident even in the raw data, without the use of CLEAN to improve the image quality. The noise near the central component is due to stationary telescope point spread function, and appears also in similar form in the calibrator ACF. (c) Recovered ACF of WR 138, imaged in the He II line at $467.7 \mathrm{~nm}$, where the WR star produces strong emission. The secondary is again evident at a lower signal-to-noise ratio. Contours at $-2 \%,+0.4 \%, 0.5 \%$, $0.6 \%, 0.7 \%, 0.8 \%, 0.9 \%, 1 \%, 2 \%, 4 \%, 8 \%, 16 \%$, and $32 \%$ of the maximum. The magnitude difference here is $5.3 \pm 0.3$ mag. The $2 \%$ negative "bowl" around the central component was caused by seeing mismatches between source and calibration star.

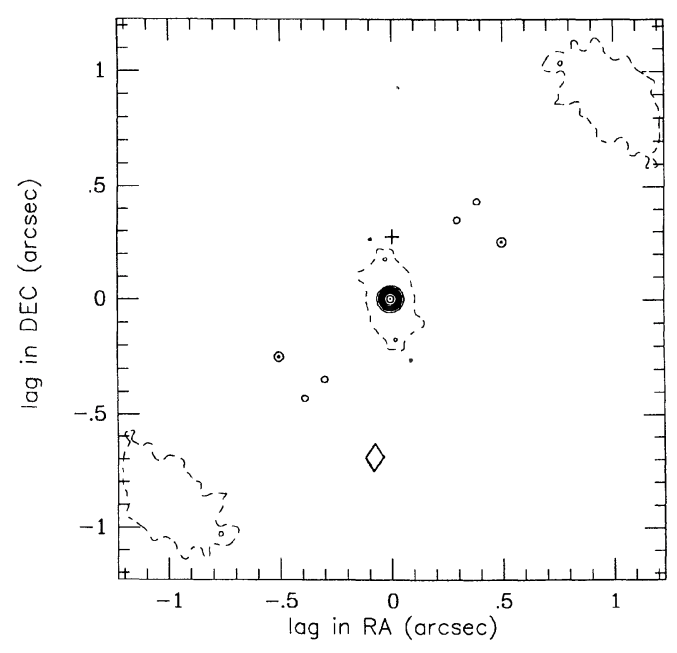

FIG. 2. $800 \mathrm{~nm}$ recovered ACF of T Tau. A cross marks the position of the $\mathrm{T}$ Tau $\mathrm{N}$ in 1984 , and a diamond marks the position of T Tau S in 1990. Contours are in logarithmic intervals, at $\pm 0.5 \%, 1 \%, 2 \%, 4 \%, 8 \%, 16 \%, 32 \%, 64 \%$, and $99 \%$ of the maximum value. 


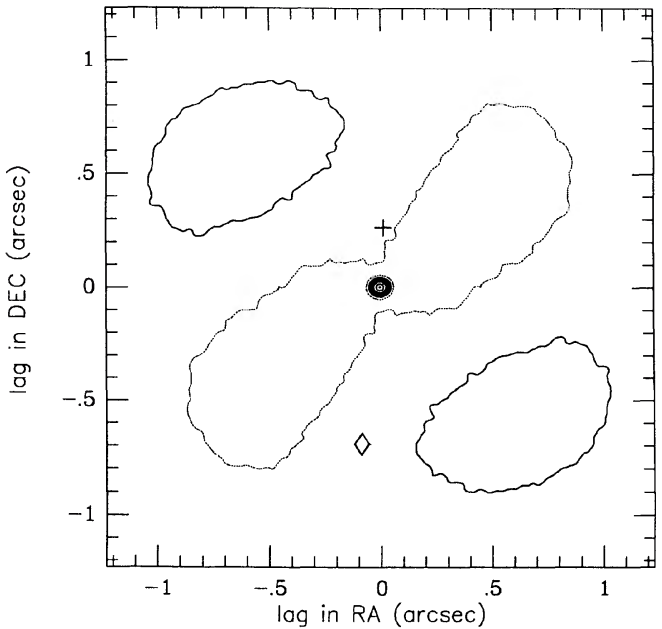

Fig. 3. $650 \mathrm{~nm}$ recovered $\mathrm{ACF}$ of $\mathrm{T}$ Tau, with positions marked as in Fig. 2. Contours are in logarithmic intervals at $\pm 1 \%, 2 \%, 4 \%, 8 \%, 16 \%, 32 \%, 64 \%$, and $99 \%$ of the maximum value. The noise is dominated by a low spatial frequency saddle, due to slight seeing mismatches between source and calibrator.

O and T Tau S have spectra with significant infrared excesses (Ghez et al. 1991), we searched all maps out to $4.8 \mu \mathrm{m}$ for any evidence of $\mathrm{T}$ Tau $\mathrm{N}$. In none of the maps was there any suggestion of a third stellar component in any part of the maps. As the results in Table 2 show, our $K$-band limits are inconsistent with constant brightness for $\mathrm{T}$ Tau $\mathrm{N}$ as reported by Maihara \& Kataza (1991) for T Tau N in 1988.

A complete set of diffraction-limited images from 1 to 5 $\mu \mathrm{m}$ of the T Tau system is presented in Ghez et al. (1991). Here we present only the $H$-band ACF (Fig. 5) which gives the most stringent limit on the presence of $\mathrm{T}$ Tau $\mathrm{N}$ and also establishes the high sensitivity of the observations. These $H$ -

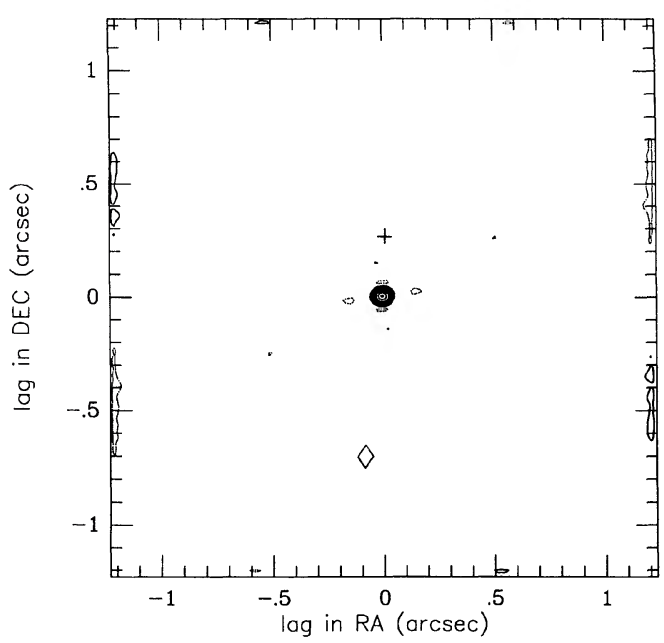

FIG. 4. Highpass filtered $650 \mathrm{~nm}$ ACF of T Tau, with contour levels at $\pm 0.5 \%, 1 \%, 2 \%, 4 \%, 8 \%, 16 \%, 32 \%, 64 \%$, and $99 \%$ of the maximum value. The filter passed spatial frequencies above $\sim 3$ cycles per ".
TABLE 2. Magnitude difference limits on T Tau N and T Tau S in 1990.

\begin{tabular}{lccc}
\hline \hline $\begin{array}{l}\text { Wavelength } \\
(\mathrm{nm})\end{array}$ & $\begin{array}{c}\mathrm{T} \mathrm{Tau} \mathrm{N}^{\mathrm{a}} \\
0.03-1.2^{\prime \prime} \\
(\mathrm{mag})\end{array}$ & $\begin{array}{c}\text { T Tau N } \\
1984 \text { position } \\
(\mathrm{mag})\end{array}$ & $\begin{array}{c}\text { T Tau S } \\
1989 \text { position } \\
(\mathrm{mag})\end{array}$ \\
\hline $650 \quad>3.5$ & $>4.0$ & $>4.2$ \\
650 (HP filtered) & $>5.4$ & $>5.4$ & $>5.6$ \\
800 & $>4.7$ & $>5.1$ & $>5.5$ \\
$1600(\mathrm{H}$ band $)$ & $>4.9$ & $>5.2$ & $\mathrm{c}$ \\
$2200(K$ band $)$ & $>5.7$ & $>5.1$ & $\mathrm{c}$
\end{tabular}

${ }^{a}$ Limits are the minimum magnitude difference allowed for any pointlike component with respect to the optical primary star ( $T$ Tau $O$ in the text), at a level corresponding to five times the rms noise in the ACF.

${ }^{b}$ Limits are the minimum magnitude difference allowed, with respect to the optical primary star, at the $95 \%$ confidence level, for a limited region near the 1984 position of the putative T Tau N. The limit is taken from a level $2 \sigma$ above the highest value within $\sim 0.08^{\prime \prime}$ of the 1984 position.

${ }^{\mathrm{c}}$ Variable; see Ghez et al. (1991).

band observations were made prior to the 1989 flare of T Tau $S$ reported by Ghez et al. (1991), when T Tau S was $\sim 5$ mag fainter than $\mathrm{T}$ Tau $\mathrm{O}$ in the $H$ band. The lowest contour in Fig. 5 is at the $0.6 \%$ level, corresponding to the $3 \sigma$ level in the ACF. T Tau S is detected at the $5 \sigma$ level here. There is no other significant feature in the map other than an artifact at the $\sim 1 \%$ level which appears as a smearing along the $x$ axis near the central peak. This artifact is well understood; it is due to two bad rows at the edge of the detector pixel array. It is correctable by interpolation over the bad rows which do not view the object in any case. The interpolation is somewhat worse in this particular instance than in a typical recovered infrared ACF. The large magnitude difference between $\mathrm{T}$ Tau $\mathrm{O}$ and $\mathrm{T}$ Tau $\mathrm{S}$ seen here $(\Delta m \sim 4.8 \mathrm{mag})$ is consistent with that expected from extrapolation of the results of $\mathrm{Ghez}$ et al., in the $J, K$, and $L$ infrared bands, and is indicative of

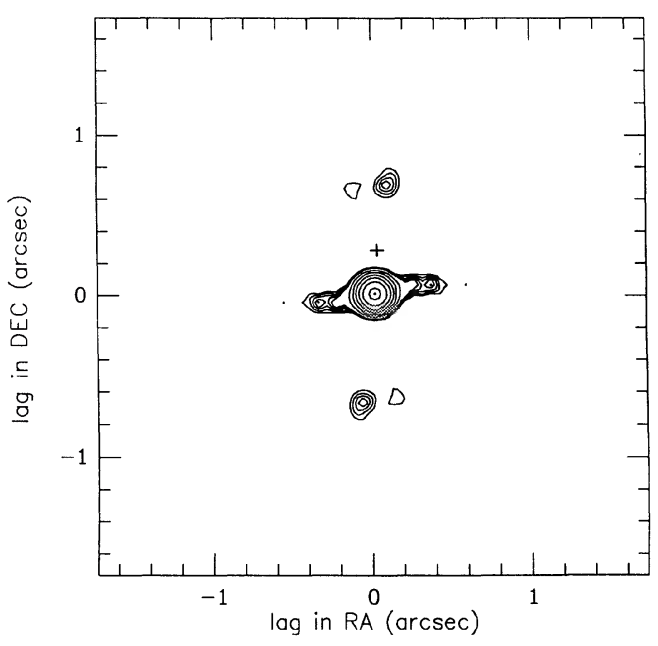

FIG. 5. $H$-band ( $1.6 \mu \mathrm{m}$ ) infrared ACF of T Tau, showing both $\mathrm{T}$ Tau $\mathrm{O}$ and the infrared companion star $\mathrm{T}$ Tau $\mathrm{S}$. The lowest contour begins at 3 times the rms noise in the ACF, at $0.6 \%$ of the maximum. The contours continue linearly at $0.5 \sigma$ intervals: $0.7 \%, 0.8 \%, 0.9 \%$, and $1.0 \%$; then logarithmically at $2 \%, 4 \%, 8 \%, 16 \%, 32 \%, 64 \%$, and $95 \%$ of the maximum. The cross again marks the position of $\mathrm{T}$ Tau $\mathrm{N}$ in 1984. The horizontal stripe near the center of the ACF is due to a detector artifact discussed in the text. 
the practical level of sensitivity of the techniques we have employed.

\subsection{Derived Limits}

The limits on the magnitude differences for all of the observations are given in Table 2. Two different limits are quoted for each wavelength band. These are given as the minimum allowable magnitude differences of $\mathrm{T}$ Tau $\mathrm{N}$ with respect to $\mathrm{T}$ Tau $\mathrm{O}$. The first limit is the $5 \sigma$ limit for the entire ACF out to radial lags of $\pm 1.2^{\prime \prime}$, where $\sigma$ is the measured rms of the noise in the ACF. This limit is typically about $2 \sigma$ above the highest noise peak in the ACFs, and represents the minimum brightness (relative to $\mathrm{T} T \mathrm{Tau} O$ ) a pointlike secondary component would be required to have to be considered detected.

The second limit is the $2 \sigma$ confidence level limit above the highest noise peak within the given angular radius around the position of T Tau N in 1984. This limit is confined to an angular radius within which $\mathrm{T}$ Tau $\mathrm{N}$ should be found if it were a star bound to $\mathrm{T}$ Tau $\mathrm{O}$, and is described in more detail in the discussion section below.

Table 2 also includes visual limits on the brightness of $\mathrm{T}$ Tau $S$ relative to $T$ Tau $O$, using the position derived by Ghez et al. (1991), and a method similar to that used for obtaining the limit for T Tau N for its 1984 location. The lack of detection of T Tau S in the $800 \mathrm{~nm} \mathrm{ACF}$ is not surprising; a blackbody extrapolation of the results of Ghez et al. indicate that the magnitude difference expected with respect to $\mathrm{T}$ Tau $\mathrm{O}$ at $800 \mathrm{~nm}$ is likely to be $>6 \mathrm{mag}$.

\section{DISCUSSION}

This section first addresses the question of the angular region which must be searched around $T$ Tau if the tertiary star is assumed bound to the system. Following this is an evaluation of how the true spectral energy distribution of an $\mathbf{M}$ dwarf compares to a blackbody, and the consequences of this for the search. Finally, a number of possible astrophysical reasons for our failure to detect $\mathrm{T} T \mathrm{Tau} \mathrm{N}$ are considered.

\subsection{Projected Motion for a Bound Object}

The maximum change $\Delta s_{\max }$ in projected angular separation over a time $\Delta t$ that is possible for a binary system where the primary is significantly more massive than the secondary can be obtained by assuming that the true separation is equal to the observed separation, and that the secondary is on a radial trajectory at just below the escape velocity. For these assumptions, the result can be written

$$
\Delta s_{\max }=\Delta t D_{*}^{-3 / 2} s_{0}^{-1 / 2}\left(2 G \mathscr{M}_{\text {primary }}\right)^{1 / 2},
$$

where $s_{0}$ is the initial angular separation, $D_{*}$ the distance to the system, and $\mathscr{M}_{\text {primary }}$ the mass of the dominant star (T Tau $O$ in our case). Equation (1) is only an approximation for $\mathrm{T} T \mathrm{Tau}$ as a triple system; however, the projected distance of $\mathrm{T} \mathrm{Tau} \mathrm{S}$ from $\mathrm{T}$ Tau $\mathrm{N}$ is more than three times that of $\mathrm{T}$ Tau $\mathrm{O}$ and $\mathrm{T}$ Tau N, so the approximation appears to be justified. For the parameters reported for the system [Nisenson et al. (1985) estimate masses of 2.2 and $0.3 \mathscr{M}_{\odot}$ for $\mathrm{T}$ Tau $\mathrm{O}$ and $\mathrm{T} T \mathrm{Tau} N$, respectively ], the six year gap in time allows $\Delta s_{\max }=0.066^{\prime \prime}$, just under two resolution elements in our optical ACFs, and less than 1 resolution element for our infrared data, indicating that the search loci for a bound tertiary are confined to be very near the position reported in
1985. Thus the second limit in Table 2 is the more physically interesting one for $\mathrm{T}$ Tau $\mathrm{N}$ if it is bound to $\mathrm{T}$ Tau $\mathrm{O}$.

\subsection{Corrections to the Expected Magnitude Differences}

Since the search sensitivity is limited by magnitude differences with the primary star, rather than the intrinsic magnitude of the components, it is important to evaluate the derived limits in light of the actual infrared behavior of the components.

The blackbody assumption for both $\mathrm{T}$ Tau $\mathrm{O}$ is a poor one since $\mathrm{T}$ Tau $\mathrm{O}$ has an infrared excess of $\sim 1.2 \mathrm{mag}$ at $2.2 \mu \mathrm{m}$ over the expected K0 star extrapolation (Ghez et al. 1991). Since molecular absorption lines dominate the continuum spectra of $M$ dwarfs in the visual and near infrared, infrared excesses also tend to be the rule rather than the exception for these stars. For example, Tinney et al. (1992) find that a sample of $\sim 20$ extreme $M$ dwarfs typically exceeded the expected blackbody values of $R-I$ by $1 \mathrm{mag}$, and exceeded the blackbody $R-K$ values by as much as 2 mag. Thus if T Tau $\mathrm{N}$ is an $\mathrm{M}$ dwarf at $3000 \mathrm{~K}$ as reported, the corrections to the infrared magnitude differences with $\mathrm{T}$ Tau $\mathrm{O}$ given above tend to be canceled out by the infrared excess that is expected for the star. In any case, it appears unlikely that the spectral peculiarities of $T$ Tau $O$ could have significantly reduced our sensitivity to $\mathrm{T}$ Tau $\mathrm{N}$.

\subsection{Critiques of the Reported Infrared Detection of T Tau N}

The results of Maihara \& Kataza (1991), which report T Tau $\mathrm{N}$ to fall on a blackbody line at $K$, imply that the star is very unusual for its class. Their derived brightness of T Tau $\mathrm{N}$ was based on a model fitting the relative brightnesses of a triple-star system to the one-dimensional averaged Fourier power spectrum. We have four separate critiques of this result.

(1) Three independent power spectra of $\mathrm{T}$ Tau $\mathrm{N}$ were averaged by Maihara \& Kataza (1991) before performing the model fit. Although the variations between the three spectra shown are well in excess of the estimated statistical errors, the errors used for the averaged spectrum did not reflect this additional variance.

(2) Although a three component model was fit, the recovered map of the system in which $\mathrm{T}$ Tau $\mathrm{N}$ appears most evident in fact showed four apparently significant components. Of these, $\mathrm{T}$ Tau $\mathrm{N}$ is the faintest. The brighter object between $\mathrm{T}$ Tau $\mathrm{O}$ and $\mathrm{T}$ Tau $\mathrm{S}$ in the map was ignored, both in the fit and in the discussion.

(3) Of the three recovered one-dimensional maps (from slit scans) presented by Maihara \& Kataza (1991), the two which show indications of $\mathrm{T}$ Tau $\mathrm{N}$ both had very poor chisquared values with respect to the data. The only map which appears to be a good fit showed no indication of $T$ Tau $N$.

(4) The component identified as T Tau N in the Maihara \& Kataza (1991) maps appeared displaced 0.12" north of the position given by Nisenson et al. (1985), a value which exceeds the permitted distance for a bound object. There was also no east-west information in their map, and their slit had an east-west length of $3.6^{\prime \prime}$.

Given these uncertainties, we take the Maihara \& Kataza (1991) result to be inconclusive as a confirmation of the presence of $\mathrm{T}$ Tau $\mathbf{N}$.

\subsection{Possible Reasons for Nondetection at Optical Wavelengths}

In this section we consider a range of possible astrophysical reasons for our nondetection of $T$ Tau N. The possibili- 
ties fall into two broad categories: variability in the brightness or color of the T Tau components, and variability in their positions.

\subsubsection{Variability in brightness or color}

Since the limits derived here are expressed in terms of magnitude difference with respect to $T$ Tau $O$, it remains to be determined whether $\mathrm{T}$ Tau $\mathrm{O}$ could possibly have brightened by more than 1-2 mag since 1984. Nisenson et al. (1985) did not report photometry of T Tau, but expressed their results only in terms of magnitude differences. $V$ band photometry compiled by Rydgren et al. (1984) indicates that T Tau has slowly brightened from $V \sim 10.4$ mag in 1962 to $V \sim 9.9 \mathrm{mag}$ in 1983 , with shorter timescale variations of $\sim 0.1$ mag possible. Photometry compiled from observations by the American Association of Variable Star Observers (Mattei 1991) spanning the period from 1982 to 1990 indicates a fairly constant value of $V \sim 10.0 \pm 0.1 \mathrm{mag}$. Unpublished measurements by Herbst, reported in Ghez et al. (1991), provide similar results for this period. Although no photometric standards were observed along with $\mathrm{T}$ Tau during our observations, a rough calibration of our optical system efficiency can be used to yield $R=9.0 \pm 0.3 \mathrm{mag}$, which gives $V=9.8 \pm 0.3 \mathrm{mag}$, using the $V-R$ value of $0.77 \mathrm{mag}$ given by Herbst et al. (1983). Our estimated photometry is consistent with the values current in 1989, and we thus rule out a significant change ( $>0.2 \mathrm{mag}$ ) in the visual brightness of $\mathrm{T}$ Tau $\mathrm{O}$ as a possibility for the nondetection of T Tau N.

Variation of the intrinsic brightness of T Tau N is a possibility which is not excluded in our results. However Nisenson et al. (1985) reported a consistent magnitude difference at $650 \mathrm{~nm}$ for observations separated by almost $1 \mathrm{yr}$. This appears to reduce the likelihood of a large amplitude periodic variation, but stochastic variations over longer timescales are possible; in fact Ghez et al. (1991) report a 2 mag flare on $T$ Tau $S$ in 1989. Given the past history of variability in $T$ Tau $\mathrm{O}$, and the recent activity in $\mathrm{T}$ Tau $\mathrm{S}$, a significant dimming of $\mathrm{T}$ Tau $\mathrm{N}$ is a possible explanation for the results we present here.

Another possibility is that the color of $\mathrm{T}$ Tau $\mathrm{N}$ has changed significantly, rather than its intrinsic brightness. However, since the spectral maximum of an object at $\sim 3000$ $\mathbf{K}$ is bracketed by the wavelengths of the four bands observed, a moderate color change should cause a relative brightening in at least one of the bands. If the emission is blackbody in nature, a moderate color change $(\sim \pm 1000$ $\mathrm{K}$ ) without a change in intrinsic luminosity is thus ruled out by our observations.

\subsubsection{Change in brightness due to variable extinction}

The infrared observations probably also rule out a moderate change in visual extinction which affects both $\mathrm{T}$ Tau $\mathrm{N}$ and $T$ Tau $O$. An increase of 2 mag of extinction in the visual implies only $0.2 \mathrm{mag}$ at $K$, using the average extinction curve given by Savage \& Mathis (1979). However, we require $\sim$ 1.5-2 mag additional extinction to T Tau at $K$ to reduce the flux density of $\mathrm{T}$ Tau $\mathrm{N}$ below our minimum detectable level. Such a change was not seen in the observations of $T$ Tau O of Ghez et al. (1991).

A change in extinction which affects only $T$ Tau $N$ is, however, quite possible in this system, due to the possible presence of a relatively dense Keplerian disk surrounding $T$ Tau. This 12 " diameter disk is reported by by Weintraub et al. (1987, 1989) from mm-wave CO observations. Weintraub et al. (1989) derive neutral hydrogen densities for the disk which fall in the range $8-20 \times 10^{5} \mathrm{~cm}^{-3}$, and ${ }^{13} \mathrm{CO}$ column densities of $2-6 \times 10^{16} \mathrm{~cm}^{-2}$. Such densities are adequate to produce visual extinctions of $10-30 \mathrm{mag}$, and $K$ band extinctions of $\sim 1-3 \mathrm{mag}$, assuming that the ratio of dust/CO in the disk is comparable to that observed in other compact clouds (cf. Spitzer 1978). Thus T Tau N may have been occulted by a dense clump of the disk material.

The large density contrast required of the clumps of disk material at the radius of $\mathrm{T}$ Tau $\mathrm{N}$ is perhaps not unexpected if the disk is tidally perturbed at the orbit of $T$ Tau $S$ as expected by the effects of Lindblad resonance. At the projected radius of $\mathrm{T}$ Tau $\mathrm{N}$ from $\mathrm{T}$ Tau $\mathrm{O}$, the Keplerian velocity is $\sim 7 \mathrm{~km} \mathrm{~s}^{-1}$, and a clump of disk material $1 \mathrm{AU}$ in diameter has an 8 month crossing time. In the absence of more detailed modeling of the effects of tidal disruption on a dense circumbinary disk, all we are able to say at present is that the required time and spatial scales do not appear to be implausible.

\subsubsection{Variability in position}

The detection of the object in November 1983 and October 1984 by Nisenson et al. (1985) indicates that the differential motion of the source was not in excess of $\sim 0.1^{\prime \prime}$ $\mathrm{yr}^{-1}$. It thus appears unlikely that the object detected by Nisenson et al. was a foreground or background object since a proper motion of $\sim 0.2^{\prime \prime} \mathrm{yr}^{-1}$ is required for it to leave our field of view. At the distance of T Tau, this implies a projected differential velocity of $250 \mathrm{~km} \mathrm{~s}^{-1}$, extremely high for a Population I star. Alternatively, a projected velocity of $\sim 40$ $\mathrm{km} \mathrm{s}^{-1}$ is required to close to within 20 mas of T Tau O, where it could not be resolved. Either projected velocity exceeds the escape velocity of the system implied by Eq. (1) by at least a factor of $\sim 4$, and the projected mean velocity of stars in the Taurus cloud by a factor of $\sim 3$.

\subsubsection{A Herbig-Haro object?}

Since the object seen by Nisenson et al. (1985) appeared brightest in a filter passband which included $\mathrm{H} \alpha$ emission, it is possible that the object reported was an emission line source. This possibility is considered by Nisenson $\mathrm{et} \mathrm{al}$. with respect to jet emission, or reflection nebulosity from emission by the central star, but the hypothesis is rejected because the apparent luminosity of the source is judged to be too high. Herbig-Haro objects (HH) (see Schwartz 1983 for a review) can appear pointlike in seeing-limited optical images, produce strong $\mathrm{H} \alpha$ emission, and are known to be variable by factors of 2-6 or more on timescales of years or less. They are associated with $\mathrm{T}$ Tauri stars, and some have proper motions which suggest ejection from the central star at velocities of hundreds of kilometers per second. The apparent visual magnitude of the object seen by Nisenson et al. in the "continuum," under the HH assumption, would be 14-15 mag. This value would put it among the brightest $\mathrm{HH}$ objects, but it it would also be among the closest to the source of excitation. In fact a number of $\mathrm{HH}$ objects are known in the vicinity of T Tau itself. Schwartz (1975) has shown that Burnham's nebula had a spectrum characteristic $\mathrm{HH}$ objects, and reported an $\mathrm{HH}$ object near the reflection nebula NGC 1555, about $30^{\prime \prime}$ W of T Tau. Recently, Schwartz (1990) has reported another HH object on the southern edge of Burnham's nebula, about 15" S of T Tau. 
Our observations at $650 \mathrm{~nm}$, with a $7.4 \mathrm{~nm}$ FWHM bandpass, would probably not have included any $\mathrm{H} \alpha$ emission, since the required radial velocity is $-1500 \mathrm{~km} \mathrm{~s}^{-1}$, too high for a $\mathrm{HH}$ object. In contrast, the observations by Nisenson et al. (1984) which used bandpasses ranging from 24 to $74 \mathrm{~nm}$, would have included many emission lines in addition to $\mathrm{H} \alpha$, including strong lines of $\mathrm{N}$ II $(658.3 \mathrm{~nm}), \mathrm{S}$ II $(671.7,673.1$ $\mathrm{nm}$ ). The $521 \mathrm{~nm}$ observations by Nisenson et al. also could include strong emission of Fe II $(515.8,526.1,527.3 \mathrm{~nm})$, and $\mathrm{N}_{\mathrm{I}}(519.8 \mathrm{~nm})$. At $450 \mathrm{~nm}$ a large number of lines of $\mathrm{Ca}$, $\mathrm{N}$, and $\mathrm{Fe}$, among others, would be included with the $50 \mathrm{~nm}$ bandpass used; these lines would, however, be on average considerably weaker than those observed in the red. It thus appears that an emission line source such as a Herbig-Haro object could account for the detection by Nisenson et al. It could also account for our lack of detection, either through variability, a large proper motion, or the narrower bandpasses used in our subsequent experiment. However, since it requires that the object be both the closest and among the brightest $\mathrm{HH}$ objects yet seen, we consider this a less likely possibility than that of either intrinsic variability or varying extinction to the source.

\section{CONCLUSIONS}

We find no evidence in 1990 September for a third stellar component associated with $\mathrm{T}$ Tauri and its southern infrared companion. If the object seen by Nisenson et al. (1985) is a star, it has either varied significantly in apparent magnitude since its discovery, or is presently in conjunction with the optical primary, thus preventing its detection. If the star is bound to the $T$ Tau system, then a significant dimming of $T$
Tau $\mathbf{N}$ is the most likely explanation for our lack of detection, since its position could not have changed more than $\sim 0.06$ " between 1984 and 1990 while remaining bound to $T$ Tau. Given the gas densities in the local environment of $T$ Tau, which may be quite high due to the presence of a bound Keplerian disk, occultation of the component by a clump of disk material is a possibility. The large density contrast required for the intervening clump could be caused by tidal disruption of the disk material by the orbit of T Tau S.

An alternative explanation is that the object seen was an emission line source such as a Herbig-Haro object, rather than a companion star. In this case, all of the observations could be explained, given the known properties of such objects. To confirm this hypothesis, future searches will require careful choice of filters and passbands to accurately measure both the continuum and possible emission lines.

We thank the staff at Palomar observatory for their invaluable help with the observations; particularly Juan Carrasco, the 200 in. telescope operator. The optical camera was graciously provided by the W. M. Keck foundation, and we thank Fernando Lopez and Richard Borrup for their assistance and advice in modifying it. We are indebted to Tom Prince and the Caltech Concurrent Supercomputing Facility for providing time on the Caltech NCUBE supercomputer and the National Science Foundation for providing support for the construction and maintenance of the infrared array camera. We thank Dr. Janet Mattei of the AAVSO for kindly providing visual photometry. S. Kulkarni's research is supported by a Presidential Young Investigator award from the National Science Foundation, and the Packard foundation. C. Haniff is supported by a SERC/NATO research fellowship.

\section{REFERENCES}

Dyck, H. M., Simon, T., \& Zuckerman, B. 1982, ApJ, 255, L103

Ghez, A. M., Neugebauer, G., Gorham, P. W., Haniff, C. A., Kulkarni, S. R.,Matthews, K., Koresko, C., \& Beckwith, S. 1991, AJ, 102, 2066

Gorham, P. W., Ghez, A. M., Haniff, C. A., \& Prince, T. A. 1990, AJ, 100, 294

Gorham, P. W., Ghez, A. M., Kulkarni, S. R., Nakajima, T., Neugebauer, G., Oke, J. B., \& Prince, T. A. 1989, AJ, 98, 1783

Gorham, P. W., Ghez, A. M., Haniff, C. A., Kulkarni, S. R., Matthews, K., \& Neugebauer, G. 1992, in preparation

Herbst, W., Holtzman, J. A., \& Klasky, R. S. 1983, AJ, 88, 1648

Hogbom, J. A. 1974, A\&AS, 15, 417

Lohmann, A. W., Weigelt, G., \& Wirnitzer, B. 1983, Appl. Opt., 22, 4028

Maihara, T., \& Kataza, H. 1991, A\&A, 249, 392

Mattei, J. 1991, American Association of Variable Star Observers (unpublished)

Moffat, A. F. J., Lamontagne, R., Shara, M. M., \& McAlister, H. A. 1986, AJ, 91, 1392

Nakajima, T., Kulkarni, S. R., Gorham, P. W., Ghez, A. M., Neugebauer,

G., Oke, J. B., Prince, T. A., \& Readhead, A. C. S. 1989, AJ, 97, 1510

Nisenson, P., Stachnik, R. V., Karovska, M., \& Noyes, R. 1985, ApJ, 297,
L17

Rydgren, A. E., Schmelz, J. T., Zak, D. S., \& Vrba, F. J. 1984, Publ. U. S. Naval Observatory, Sec. Ser. Vol. XXV, P. 1

Savage, B. D., \& Mathis, J. S. 1979, ARA\&A, 17, 73

Schwartz, R. D. 1975, ApJ, 195, 631

Schwartz, R. D. 1983, ARA\&A, 21, 209

Schwartz, P. R., Simon, T., Zuckerman, B., \& Howell, R. R. 1984, ApJ, 280, L23

Schwartz, P. R., Simon, T., \& Campbell, R. 1986, ApJ, 303, 233

Schwartz, R. D. 1990, in Flare Stars in Star Clusters, Associations, and the Solar Vicinity, IAU Colloquium No. 137, edited by L. V. Mirzoyan et al., p. 221

Spitzer, Jr., L. 1978, Physical Processes in the Interstellar Medium (Wiley, New York), pp. 163

Tinney, C. G., Mould, J. R., \& Reid, I. N. 1992, AJ (in press)

van der Hucht, K. A., Conti, P. S., Lundstrom, I., \& Stenholm, B. 1981, SSRV, 28, 227

Weintraub, D. A., Masson, C. R., \& Zuckerman, B. 1987, ApJ, 320, 336

Weintraub, D. A., Masson, C. R., \& Zuckerman, B. 1989, ApJ, 344, 915 